\title{
Identifying new variables during infection: proximity to the host epithelium and epigenetic programs alter the expression of virulence factors in Vibrio cholerae
}

\author{
Benjamin Mudrak and Rita Tamayo* \\ Department of Microbiology and Immunology, University of North Carolina at Chapel Hill School of Medicine, Chapel Hill, NC, USA \\ *Correspondence: rtamayo@med.unc.edu
}

In the life cycles of facultative pathogens, the ability to transition between the external environment and the host is key to survival. These transitions are accompanied by alterations in transcriptional and proteomic profiles (Merrell et al., 2002; Schild et al., 2007; LaRocque et al., 2008). Numerous studies have investigated the changes in gene expression that occur during these environmental shifts and have identified factors that are important for survival and growth in the two disparate settings. Less is known about the changes in gene expression that result as a pathogen transitions between microenvironments within the host or even the number of such transitions that occur. A single organ or tissue may in fact constitute a wide array of strikingly different environmental conditions for an invading pathogen.

Many studies have also assumed a homogeneous response on the part of the population of pathogenic cells, but increasing evidence suggests that bacterial cells can specialize, producing two stable phenotypes within a single isogenic population using an epigenetic mechanism called bistability (Veening et al., 2008). Recently, the presence of bistable subgroups has been noted in Bacillus subtilis biofilms (Chai et al., 2008) and in cultures of Pseudomonas aeruginosa (Turner et al., 2009) and Porphyromonas gingivalis (Suwannakul et al., 2010). In B. subtilis, key sporulation genes are differentially expressed within isogenic subpopulations in a biofilm. In $P$. aeruginosa and $P$. gingivalis, certain virulence factors exhibit bistable regulation. One hallmark of bistability is the transience of the phenotype; cells in either subgroup give rise to cells of both types after further passaging. The existence of two populations has been likened to "bet-hedging," with bacteria in bistable populations ready to thrive under more conditions than a homogeneous population (Veening et al., 2008).
Vibrio cholerae is the Gram-negative bacterium responsible for outbreaks of the deadly diarrheal disease cholera (Kaper et al., 1995). These outbreaks occur across the globe, and mortality rates can be considerable when proper therapy, usually oral rehydration, is not available. The primary virulence factors produced by $V$. cholerae are cholera toxin (CT), which is responsible for generating the massive efflux of fluid and electrolytes that culminates in diarrhea, and the toxin-coregulated pilus (TCP), a bundle-forming pilus required for colonization of the host intestine. Although the complex regulatory mechanisms controlling $\mathrm{CT}$ and TCP production have been defined in great detail, uncovering the dynamics of virulence factor expression during infection would improve our understanding of $V$. cholerae pathogenesis and transmission.

In a recent study, Nielsen et al. (2010) have provided strong evidence that two populations of $V$. cholerae cells arise during infection. Using the rabbit ileal loop model, the authors showed that the transcriptional profile of $V$. cholerae cells changes significantly based on the proximity of the cells to the intestinal epithelium. Taking advantage of a clever reporter system, transient expression of the tcp locus, which encodes the system required for TCP biogenesis, could be tracked by measuring bacterial fluorescence. Bacteria localized to the intestinal surface demonstrated much higher fluorescence, and thus higher tcp expression, than bacteria in the lumen. Similarly, expression of $r r n B$, a locus regulated by growth rate, was found to be highest in surface-associated bacteria, indicating that the bacteria near the host epithelium replicate more rapidly. Further studies showed that there were two populations of bacteria at late stages of infection: one group of cells continuing to express $t c p$ and another group not expressing tcp. Interestingly, the tcp-expressing cells were frequently found in aggregates, consistent with the role of TCP in microcolony formation in V. cholerae (Kirn et al., 2000). In vitro experiments demonstrated that these subpopulations were bistable and that the tcp expression phenotype could be induced with bicarbonate, a compound naturally released by the intestine to neutralize its contents (Kiela and Ghishan, 2009). More importantly, the bifurcation of TCP expression could be detected in vivo, after infection of rabbit ileal loops.

It has been shown previously that $V$. cholerae passaged through a host or grown in a biofilm in vitro becomes transiently hyperinfectious compared to isogenic bacteria grown in shaking culture (Merrell et al., 2002; Tamayo et al., 2010). Another study has demonstrated that clumps of $V$. cholerae are shed after host passage and that these "in vivo-induced biofilms" display greater hyperinfectivity than free-living bacteria that exit the human host (Faruque et al., 2006). Thus, biofilms or aggregates formed in vitro and in vivo are both potentially primed for colonization of the host gastrointestinal tract. As noted by Nielsen et al. (2010) it is tempting to speculate that the TCP-positive aggregates observed in their study may be related to the hyperinfectious aggregates shed by infected patients. Prior expression of TCP by host-passaged bacteria may facilitate attachment or microcolony formation in the small intestine.

Certainly, much work remains to be done to ascertain the signals influencing V.cholerae in different microenvironments within and outside the host. However, in their recent work and in an earlier report detailing the role of RpoS in the V. cholerae mucosal escape response (Nielsen et al., 2006), the Schoolnik lab has highlighted some added complications to the study of bacterial infection. Infections were once thought of in terms of early and late timepoints and location within specific organs (such as the small intestine for V. cholerae). It is now apparent that even cells 
found in the same time and place (such as $V$. cholerae shed by a host) may represent multiple, distinct subpopulations, one or both of which may be responsible for previously observed phenotypes.

Looking beyond $V$. cholerae pathogenesis, epigenetic control of virulence factors may underlie other cases of transient infectivity. Indeed, a recent report detailing a bistable epigenetic response to bicarbonate in Salmonella enterica indicates that such programs may be widespread, at least among enteric pathogens (Kroger et al., 2011). Although it poses significant challenges, it is clearly important to consider variables specific to different microenvironments (e.g., the lumen or epithelial surface) and different subpopulations of bacteria within those same spatiotemporal domains.

\section{REFERENCES}

Chai, Y., Chu, F., Kolter, R., and Losick, R. (2008). Bistability and biofilm formation in Bacillus subtilis. Mol. Microbiol. 67, 254-263.

Faruque, S. M., Biswas, K., Udden, S. M., Ahmad, Q. S., Sack, D. A., Nair, G. B., and Mekalanos, J. J. (2006). Transmissibility of cholera: in vivo-formed biofilms and their relationship to infectivity and persistence in the environment. Proc. Natl. Acad. Sci. U.S.A. 103, 6350-6355.
Kaper, J. B., Morris, J. G. Jr., and Levine, M. M. (1995). Cholera. Clin. Microbiol. Rev. 8, 48-86.

Kiela, P. R., and Ghishan, F. K. (2009). Ion transport in the intestine. Curr. Opin. Gastroenterol. 25, 87-91.

Kirn, T. J., Lafferty, M. J., Sandoe, C. M., and Taylor, R. K. (2000). Delineation of pilin domains required for bacterial association into microcolonies and intestinal colonization by Vibrio cholerae. Mol. Microbiol. 35, 896-910.

Kroger, C., Srikumar, S., Ellwart, J., and Fuchs, T. M. (2011). Bistability in myo-inositol utilization by Salmonella enterica serovar Typhimurium. J. Bacteriol. 193, 1427-1435

LaRocque, R. C., Krastins, B., Harris, J. B., Lebrun, L. M., Parker, K. C., Chase, M., Ryan, E. T., Qadri, F., Sarracino, D., and Calderwood, S. B. (2008). Proteomic analysis of Vibrio cholerae in human stool. Infect. Immun. 76, 4145-4151.

Merrell, D. S., Butler, S. M., Qadri, F., Dolganov, N. A., Alam, A., Cohen, M. B., Calderwood, S. B., Schoolnik, G. K., and Camilli, A. (2002). Host-induced epidemic spread of the cholera bacterium. Nature 417, 642-645.

Nielsen, A. T., Dolganov, N. A., Otto, G., Miller, M. C., Wu, C. Y., and Schoolnik, G. K. (2006). RpoS controls the Vibrio cholerae mucosal escape response. PLoS Pathog. 2, e109. doi: 10.1371/journal.ppat.0020109

Nielsen, A. T., Dolganov, N. A., Rasmussen, T., Otto, G., Miller, M. C., Felt, S. A., Toreilles, S., and Schoolnik, G. K. (2010). A bistable switch and anatomical site control Vibrio cholerae virulence gene expression in the intestine. PLoS Pathog. 6, el001102. doi: 10.1371/ journal.ppat.1001102

Schild, S., Tamayo, R., Nelson, E. J., Qadri, F., Calderwood, S. B., and Camilli, A. (2007). Genes induced late in infection increase fitness of Vibrio cholerae after release into the environment. Cell Host Microbe 2, 264-277.

Suwannakul, S., Stafford, G. P., Whawell, S. A., and Douglas, C. W. (2010). Identification of bistable populations of Porphyromonas gingivalis that differ in epithelial cell invasion. Microbiology 156, 3052-3064

Tamayo, R., Patimalla, B., and Camilli, A. (2010). Growth in a biofilm induces a hyperinfectious phenotype in Vibrio cholerae. Infect. Immun. 78, 3560-3569.

Turner, K. H., Vallet-Gely, I., and Dove, S. L. (2009). Epigenetic control of virulence gene expression in Pseudomonas aeruginosa by a LysR-type transcription regulator. PLoS Genet. 5, e1000779. doi: 10.1371/ journal.pgen.1000779

Veening, J. W., Smits, W. K., and Kuipers, O. P. (2008). Bistability, epigenetics, and bet-hedging in bacteria. Annu. Rev. Microbiol. 62, 193-210.

Received: 22 February 2011; accepted: 23 February 2011; published online: 07 March 2011.

Citation: Mudrak B and Tamayo R (2011) Identifying new variables during infection: proximity to the host epithelium and epigenetic programs alter the expression of virulence factors in Vibrio cholerae. Front. Microbio. 2:42. doi: 10.3389/fmicb.2011.00042

This article was submitted to Frontiers in Cellular and Infection Microbiology, a specialty of Frontiers in Microbiology. Copyright (c) 2011 Mudrak and Tamayo. This is an openaccess article subject to an exclusive license agreement between the authors and Frontiers Media SA, which permits unrestricted use, distribution, and reproduction in any medium, provided the original authors and source are credited. 